\title{
Roof region dependent wind potential assessment with different RANS turbulence models
}

\author{
Francisco Toja-Silva ${ }^{\mathrm{a}, \mathrm{b}}$, Carlos Peralta ${ }^{\mathrm{c}}$, Oscar Lopez-Garcia ${ }^{\mathrm{b}}$, Jorge Navarro ${ }^{\mathrm{a}}$, \\ Ignacio $\mathrm{Cruz}^{\text {a }}$ \\ a Centro de Investigaciones Energéticas, Medioambientales y Tecnológicas (CIEMAT), Av. Complutense 40, 28040 Madrid, Spain \\ ${ }^{\mathrm{b}}$ Escuela Técnica Superior de Ingenieros Aeronáuticos, Universidad Politécnica de Madrid (UPM), Madrid, Spain \\ ${ }^{c}$ Fraunhofer IWES, Ammerlaender Heerstrasse 136, Oldenburg, Germany
}

\begin{abstract}
A B S T R A C T
The analysis of the wind flow around buildings has a great interest from the point of view of the wind energy assessment, pollutant dispersion control, natural ventilation and pedestrians wind comfort and safety. Since LES turbulence models are computationally time consuming when applied to real geometries, RANS models are still widely used. However, RANS models are very sensitive to the chosen turbulence parametrisation and the results can vary according to the application. In this investigation, the simulation of the wind flow around an isolated building is performed using various types of RANS turbulence models in the open source code OpenFOAM, and the results are compared with benchmark experimental data. In order to confirm the numerical accuracy of the simulations, a grid dependency analysis is performed and the convergence index and rate are calculated. Hit rates are calculated for all the cases and the models that successfully pass a validation criterion are analysed at different regions of the building roof, and the most accurate RANS models for the modelling of the flow at each region are identified. The characteristics of the wind flow at each region are also analysed from the point of view of the wind energy generation, and the most adequate wind turbine model for the wind energy exploitation at each region of the building roof is chosen.
\end{abstract}

\section{Introduction}

The analysis of the wind flow around buildings has a great interest from the point of view of the wind energy exploitation, pollutant dispersion control, natural ventilation (van Hooff and Blocken, 2010; Ramponi and Blocken, 2012) and pedestrians wind comfort and safety (Blocken et al., 2012). Toja-Silva et al. (2013) present a review of the opportunities and challenges of urban wind energy that stresses the necessity to perform accurate analyses of the flow behaviour on building roofs, in order to get more information about possible positions of wind turbines to take advantage of the accelerating effect of the wind above the building, the adequate kind of turbine and the estimation of the power generation. Some authors (Ledo et al., 2011; Lu and Ip, 2009; Ricciardelli and Polimeno, 2006; Ng et al., 2011; Abohela et al., 2013) have carried out similar analyses reaching the same conclusions. A careful analysis must include both experimental and numerical studies (Blocken, 2014).

As part of the urban wind energy activities at the CIEMAT, an experimental campaign of the wind flow around a building is being planned. The purpose of the experiment is to obtain precise measurements of the flow field data of a real scale building where small wind turbines can be installed on its roof, which then will be used to validate results of computational fluid dynamics (CFD) simulations. The final aim of this project is to produce a real-scale urban wind benchmark, specifically tailored for wind energy exploitation, that can be used by the urban CFD modelling community. In order to investigate possible scenarios and the placement of the measurement points, a numerical study is presented in this investigation, with the aim of identifying the best locations and types of wind turbines to be placed on the building roof.

The Direct Numerical Simulation (DNS) of the wind flow around a full-scale building geometry is unattainable. Therefore, some simplifying assumptions must be made. There are two main numerical approaches to flow modelling: Large-Eddy Simulation (LES) and Reynolds Averaged Navier-Stokes Equations (RANS). The LES consists in the modelling of the near wall flow by using space 
filtered equations (Pope, 2000; Sagaut, 2006). LES presents an agreement with experimental data better than RANS, but its computational cost is very high for full-scale geometries, especially in the case of the wind flow around buildings (Franke et al., 2007). The other main flow modelling technique consists in the solution of the RANS equations. Currently, state-of-the-art RANS modelling involves the use of two-equation closures for the purposes of wind energy resource assessment over (moderately) complex terrain. A recent comparison of models for the benchmark case of Bolund can be found in Sumner (2012).

The choice of the turbulence model is a compromise between the accuracy and the computational cost. Since the purpose of computational wind engineering (CWE) analysis is to extend the conclusions to real-scale cases, and the computational cost of LES with full-scale geometries is too expensive nowadays (Franke et al., 2007; Sumner et al., 2010), there is a necessity of a better parametrisation of turbulence models used in RANS modelling to effectively deal with real-scale cases. In agreement with this, the main objective of the present work is to determine the best 2-equation turbulence models from the point of view of the urban wind energy exploitation. To do this, some $k-\varepsilon$ and $k-\omega$ models are tested performing simulations with the free, open source CFD software package OpenFOAM (2013). In order to compare the simulation results with experimental values, the benchmark case A of the Architectural Institute of Japan (Guidebook for Practical Applications of CFD to Pedestrian Wind Environment around Buildings, 2013) is used. This benchmark case is an isolated building of aspect ratio 1:1:2 tested in a wind tunnel by Meng and Hibi (1998).

A similar study was undertaken by Tominaga et al. (2008) using both linear RANS (LK, MMK and Durbin's revised $k-\varepsilon$ ) and LES models, and by Shao et al. (2012a) using non-linear RANS models (Shih, Craft and Ehrhard). Gousseau et al. (2013) and Kono and Kogaki (2013) performed LES simulations (using the Smagorinsky model) for the same building studied in the present investigation, obtaining better agreement with experimental data at higher computational cost. This is the reason why RANS models (especially in combination with the $k-\varepsilon$ model) are widely used in industrial applications for complex cases. These previous studies (Tominaga et al., 2008; Shao et al., 2012a) analysed the whole flow (including upstream and downstream) but were not focussed on the flow behaviour on the building roof from the point of view of wind energy exploitation. Steady RANS simulations are not always in good agreement with experimental results because flow unsteadiness due to the vortex shedding behind the building is not well reproduced (Tominaga et al., 2008). In this work, we test different turbulence models, focusing on reproducing experimental measurements for both velocity and turbulence kinetic energy on the building roof. Therefore, the present investigation is aimed at bringing useful information about the accuracy of different 2-equation turbulence models for the wind energy assessment in the different regions of the building roof, since different 2-equation turbulence models have different degrees of reliability under particular flow conditions (detachment, reattachment, recirculation, etc.). Furthermore, the flow is analysed and the possibility of wind energy exploitation is commented at each region, recommending the wind turbine type more adequate for each situation.

In the following, the turbulence modelling is discussed in Section 2 and the case study description and the simulation details are presented in Section 3. Afterwards, Section 4 presents the obtained results and the discussion regarding the behaviour of RANS models and the flow at each region of the building roof from the point of view of the wind energy exploitation. Finally, conclusions and recommendations for further developments are commented in Section 5.

\section{Dynamics and turbulence modelling}

The steady-state RANS equations for an incompressible fluid without body forces can be written as (Cheng et al., 2003)

$\frac{\partial \bar{u}_{i}}{\partial x_{i}}=0$

for the mass conservation, and for the momentum conservation

$\frac{\partial\left(\bar{u}_{i} \bar{u}_{j}\right)}{\partial x_{j}}=-\frac{1}{\rho} \frac{\partial \bar{p}}{\partial x_{i}}+\frac{\partial}{\partial x_{j}}\left(\nu \frac{\partial \bar{u}_{i}}{\partial x_{j}}-\overline{u_{i} u_{j}^{\prime}}\right)$,

where $\bar{p}$ is the mean pressure and $\rho$ and $\nu$ are the fluid density and kinematic viscosity, respectively. The Reynolds stresses $\left(\overline{u_{i} u_{j}^{\prime}}\right)$ must be prescribed in terms of the mean flow values. Considering the Boussinesq linear isotropic eddy-viscosity hypothesis (linear relationship between the turbulent stresses and the mean velocity gradients), the statistical turbulence closure model yields

$-\overline{u_{i} u_{j}}=2 u_{t} S_{i j}-\frac{2}{3} k \delta_{i j}$

where

$S_{i j}=\frac{1}{2}\left(\frac{\partial \bar{u}_{i}}{\partial x_{j}}+\frac{\partial \bar{u}_{j}}{\partial x_{i}}\right)$

is the strain rate tensor, $u_{t}$ is the kinematic eddy viscosity, $\delta_{i j}$ is the Kronecker Delta function and $k=\frac{1}{2} \overline{u_{i}^{\prime} u_{i}^{\prime}}$ is the turbulent kinetic energy. The equations for the turbulent kinetic energy and the turbulence dissipation rate $(\varepsilon)$ are necessary to solve all the unknowns. These equations, in steady-state form without considering buoyancy, are

$\frac{\partial\left(\bar{u}_{j} k\right)}{\partial x_{j}}=\frac{\partial}{\partial x_{j}}\left[\left(\nu+\frac{\psi_{t}}{\sigma_{k}}\right) \frac{\partial k}{\partial x_{j}}\right]+P_{k}-\varepsilon$

and

$\frac{\partial\left(\bar{u}_{j} \varepsilon\right)}{\partial x_{j}}=\frac{\partial}{\partial x_{j}}\left[\left(\nu+\frac{u_{t}}{\sigma_{\varepsilon}}\right) \frac{\partial \varepsilon}{\partial x_{j}}\right]+C_{\varepsilon} \frac{\varepsilon}{k} P_{k}-C_{\varepsilon 2} \frac{\varepsilon^{2}}{k}$,

where $P_{k}$ is the production of $k$, and $\sigma_{k}$ and $\sigma_{\varepsilon}$ (Prandtl numbers), $C_{\varepsilon 1}$ and $C_{\varepsilon 2}$ are closure constants. The production of $k$ in the standard $k-\varepsilon$ model (SKE) is

$P_{k}=\nu_{4} S^{2}$,

where $S$ is the modulus of the rate of strain tensor and

$4=C_{\mu} \frac{k^{2}}{\varepsilon}$,

where $C_{\mu}$ is the proportional number, another constant parameter.

There are some modifications of the SKE model, developed to improve the accuracy of the results, especially the overestimation of the turbulent kinetic energy in the impinging region of bluff bodies. Launder and Kato (1993) (KL model) propose the calculation of $P_{k}$ as a function of the strain rate scale $(S)$ and the vorticity scale $(\Omega)$ :

$P_{k}=\nu_{4} S \Omega$.

However, the KL model has a mathematical inconsistency in the modelling of $-\overline{u_{i} u_{j}^{\prime}}$ and $P_{k}$, between Eqs. (3) and (9). Furthermore, the KL model overestimates $P_{k}$ (comparing with SKE) when $\Omega / S>1$. To correct these problems, Tsuchiya et al. (1997) introduced the Murakami-Mochida-Kondo (MMK) model that adds a modification to the expression for $\nu_{t}$ : 
$\nu_{t}=\frac{C_{\mu} k^{2} \Omega}{\varepsilon S}$

only applicable when $\Omega / S<1$. Otherwise, Eq. (8) is applicable, as in the SKE model.

Durbin (1996) proposed another $k-\varepsilon$ modification to correct the $k$ overestimation in the SKE model by calculating $\nu_{t}$ related to the turbulence velocity time scale $(T)$ :

$\nu_{t}=C_{\mu} k T$.

Since in the SKE model $k / \varepsilon$ is adopted for $T$, the proposed bound on the time scale of Durbin (1996) is

$T=\min \left(T_{S K E}, T_{D}\right)$,

where

$T_{S K E}=k / \varepsilon$

and

$T_{D}=\frac{1}{3 C_{\mu} S} \sqrt{\frac{3}{2}}$.

There are variations of the $T_{D}$ variable in the Durbin model. Tominaga et al. (2008) use

$T_{D}=\frac{1}{C_{\mu} S \sqrt{3}}$.

According to Durbin (1996), Eq. (14) can be modified to obtain agreement with experimental data. In the present case, we have empirically found the following expression (explained in detail in Section 4) to obtain agreement of the recirculation distance on the building roof with the experimental data:

$\mathrm{T}_{D}=\frac{32}{45 C_{\mu} S}$.

Yap (1987) also proposed a correction to the $k-\varepsilon$ model. It consists in the addition of a source term $S$, to the right hand side of epsilon equation, Eq. (6). The added source term is

$S_{\varepsilon}=0.83 \frac{\varepsilon^{2}}{k}\left(\frac{k^{1.5}}{\varepsilon_{e}}-1\right)\left(\frac{k^{1.5}}{\varepsilon_{e}}\right)^{2}$,

$l=C_{\mu}^{-0.75} \kappa y_{n}$, where $y_{h}$ is the normal distance to the nearest wall. Yap's correction is usually applied together with the KL model (called KLY) (Kato and Launder, 1993).

Yakhot and Smith (1992) developed the $k-\varepsilon$ Re-Normalisation Group (RNG) model, also by adding a term $(\mathfrak{R})$ to the right hand side of Eq. (6),

$\mathfrak{R}=\frac{C_{\mu} \eta^{3}\left(1-\frac{\eta}{\eta_{0}}\right)}{1+\beta \eta^{3}} \frac{\varepsilon^{2}}{k}$,

where $\eta=S k / \varepsilon$ and the rest of the parameters are constants of the model (Yakhot and Smith, 1992; Kim and Baik, 2004).

Besides the $k-\varepsilon$ models described previously, we also consider the $k-\omega$ shear stress transport (SST) (Menter, 1994) model. This model follows the $k-\omega$ approach at the near-wall region, and switches to the $k-\varepsilon$ away from the surface. The steady state transport equations of the $k-\omega$ SST model without source terms are

$\frac{\partial\left(\rho \bar{u}_{j} k\right)}{\partial x_{j}}=\frac{\partial}{\partial x_{j}}\left(I_{k} \frac{\partial k}{\partial x_{j}}\right)+\tilde{P}_{k}-Y_{k}$

and

$\frac{\partial\left(\rho \bar{u}_{j} \omega\right)}{\partial x_{j}}=\frac{\partial}{\partial x_{j}}\left(\Gamma_{\omega} \frac{\partial \omega}{\partial x_{j}}\right)+P_{\omega}-Y_{\omega}+D_{\omega}$,

where $\omega$ is the specific dissipation, $I_{k}$ and $\Gamma_{\omega}$ are the effective diffusivities of $k$ and $\omega$, respectively, $\tilde{P}_{k}$ is the generation of $k$ due to mean velocity gradients, $P_{\omega}$ is the generation of $\omega, Y_{k}$ and $Y_{\omega}$ are the dissipations of $k$ and $\omega$, respectively, and $D_{o}$ is the cross-diffusion term.

Additionally to the linear models described above, we also consider the Nonlinear $k-\varepsilon$ Shih (Shih et al. (1993)) model. The non-linear eddy viscosity models, in general, have been developed to improve the Boussinesq approximation adopted in the linear eddy viscosity turbulence models keeping the stability and applicability conditions. The Nonlinear $k-\varepsilon$ Shih model consists in the addition of the quadratic term to Eq. (3). The quadratic Reynolds stresses equation yields

$-\overline{u_{i} u_{j}}=2 \nu_{l} S_{i j}-\frac{2}{3} k \delta_{i j}-C_{1} \psi_{i} \frac{k}{\varepsilon}\left(S_{i k} S_{k j}-\frac{1}{3} S_{k l} S_{k l} \delta_{i j}\right)$

where $C_{1}$ is an empirical coefficient.

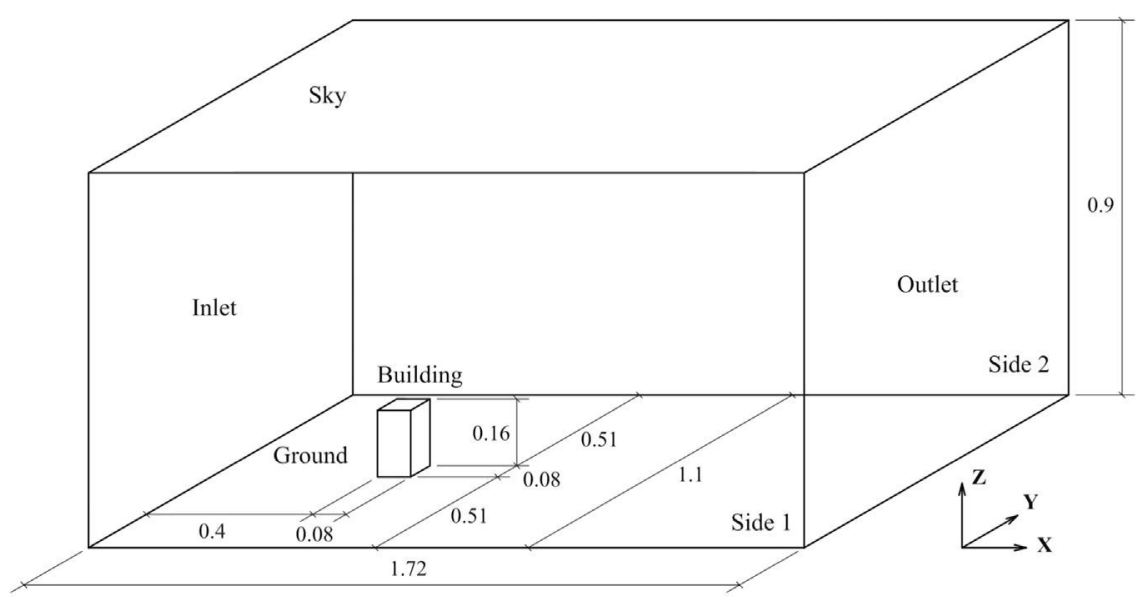

Fig. 1. Diagram of the case of study. All dimensions are in meters. 


\section{Description of the case study and simulation details}

The case study is the benchmark case A of the Architectural Institute of Japan (Guidebook for Practical Applications of CFD to Pedestrian Wind Environment around Buildings, 2013). This benchmark case is an isolated building of aspect ratio 1:1:2 placed within an atmospheric boundary layer, tested in a wind tunnel by Meng and Hibi (1998). Fig. 1 shows the geometry of the problem. Other investigations (such as Tominaga et al., 2008; Gousseau et al., 2013) describe the geometry in dimensionless units, but in the present investigation the real dimensions of the wind tunnel are specified because the results differ significatively with the domain size (especially $k$ ) due to the variation of the Re number.

We perform the simulations in OpenFOAM (2013). Table 1 presents the boundary conditions imposed for all the variables at each boundary of the simulation domain. The inlet profiles, used in the wind tunnel experiment of Meng and Hibi (1998), are shown in Fig. 2. They are set in OpenFOAM with the utility setDiscreteFields (Contrib setDiscreteFields, 2013). The wall functions are standard functions of OpenFOAM.

The steady-state simpleFoam solver for incompressible turbulent flow is used to solve the partial differential equations. For the spatial discretisation of differential operators, the Gaussian integration was used with different interpolation schemes. The 2nd order linear interpolation was applied for Gradient terms, the 2nd order upwind interpolation for Divergence terms, while for the Laplacian terms the 2nd order linear interpolation was used with explicit non-orthogonal correction (Rákai et al., 2014; Balogh

Table 1

Boundary conditions imposed at each boundary of the domain following Guidebook for Practical Applications of CFD to Pedestrian Wind Environment around Buildings (2013) and Tominaga et al. (2008). Nomenclature: iP, inlet Profile; $z G$ zeroGradient; C, Calculated; fV, fixed Value; wF, wall Function; and sP, symmetry Plane.

\begin{tabular}{llllll}
\hline Boundary & $U$ & $k$ & $\varepsilon$ & $\nu_{t}$ & $p$ \\
\hline Inlet & $\mathrm{iP}$ & $\mathrm{iP}$ & $\mathrm{iP}$ & $\mathrm{C}$ & $\mathrm{zG}$ \\
Outlet & $\mathrm{zG}$ & $\mathrm{zG}$ & $\mathrm{zG}$ & $\mathrm{C}$ & $\mathrm{fV}$ zero \\
Ground & $\mathrm{fV}$ zero & $\mathrm{kqR} w \mathrm{~F}$ & epsilon $w \mathrm{~F}$ & nutk rough $w F$ & $\mathrm{zG}$ \\
Building & $\mathrm{fV}$ zero & $\mathrm{kqR} w \mathrm{~F}$ & epsilon $\mathrm{wF}$ & nutk wF & $\mathrm{zG}$ \\
Sky & $\mathrm{fV}$ zero & $\mathrm{kqr} w \mathrm{~F}$ & epsilon $w \mathrm{~F}$ & nutk rough $w F$ & $\mathrm{zG}$ \\
Sides & $\mathrm{sP}$ & $\mathrm{sP}$ & $\mathrm{sP}$ & $\mathrm{sP}$ & $\mathrm{sP}$ \\
\hline
\end{tabular}

et al., 2012). Regarding the linear system solvers, generalised geometric-algebraic multi-grid solver (GAMG) with DIC smoother is used for the pressure, and preconditioned bi-conjugate gradient solver for asymmetric matrices (PBiCG) with diagonal incomplete (Lu and Ip, 2009) (DILU) preconditioner is used for the rest of variables. Second order accurate numerical schemes (both central differencing and upwind) must be used at least in order to avoid problems with false diffusion (Rákai et al., 2014; Balogh et al., 2012; Bakker, 2015; ANSYS Fluent, 2015).

Regarding the mesh, the external domain (inlet, outlet, ground, sky and sides in Fig. 1) is implemented using the conventional blockMesh application of OpenFOAM (Fig. 3a) with a grading of 4 in vertical direction. The building geometry, previously designed with a CAD tool and saved in STL format, is embedded into the external mesh using the snappyHexMesh application. The snappyHexMesh application is an adaptative refinement meshing utility of OpenFOAM very appropriate to mesh complex geometries, such as buildings with different shapes from stereolithography (STL) CAD files (OpenFOAM, 2013; SnappyHexMesh, 2013; SnappyHexMeshDict, 2013). This allows to deal with any building shape. The mesh around the building is refined and adapted to its shape. The application allows to define independent refinement boxes but, in the present case, the refinement is applied around the building surfaces. The refinement distance around the building surfaces is $0.16 \mathrm{~m}$ in this case. Fig. 7 shows the final mesh obtained. The total number of cells in the final mesh is close to $3.1 \mathrm{M}$.

\section{Results and discussion}

In what follows, the simulation results, together with experimental data from Meng and Hibi (1998) are presented and discussed, identifying the most appropriate models for the different regions of the building roof. Afterwards, the characteristics of the wind flow are analysed, bringing an evaluation of the possibility of the wind exploitation and the most appropriated kind of turbine at each region.

\subsection{Simulation results}

Since this investigation focuses on wind energy exploitation, we concentrate our analysis on the building roof, avoiding a
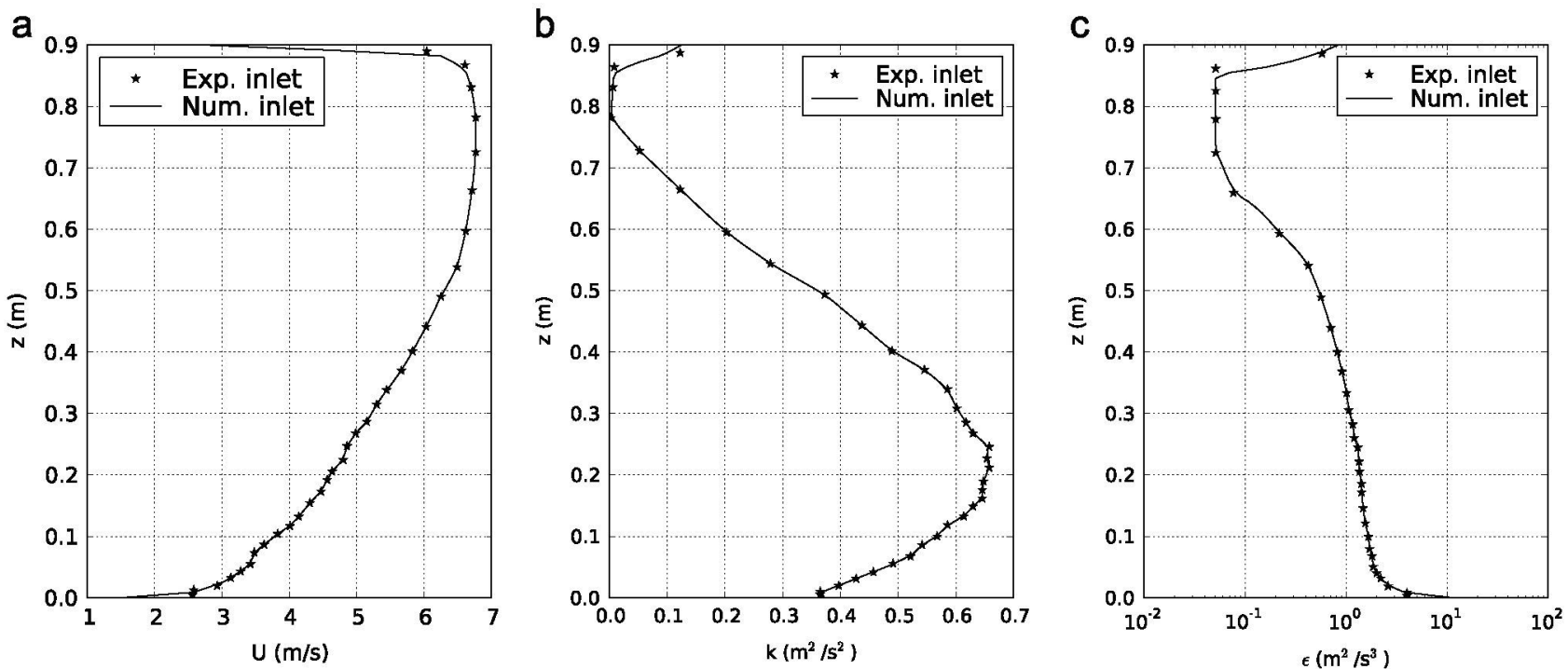

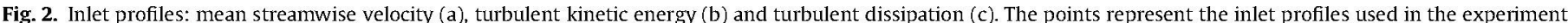
of Meng and Hibi (1998), and the solid lines are the numerical inlets of the simulations. 
a

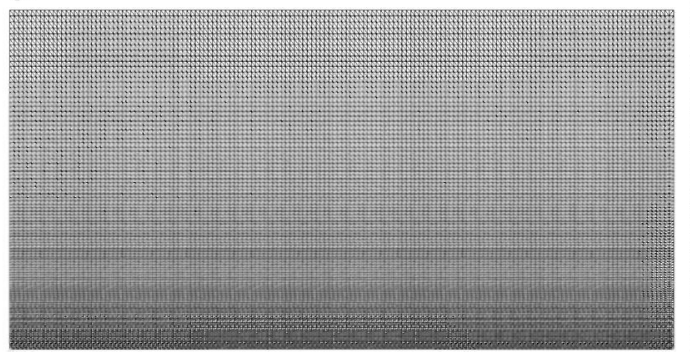

b

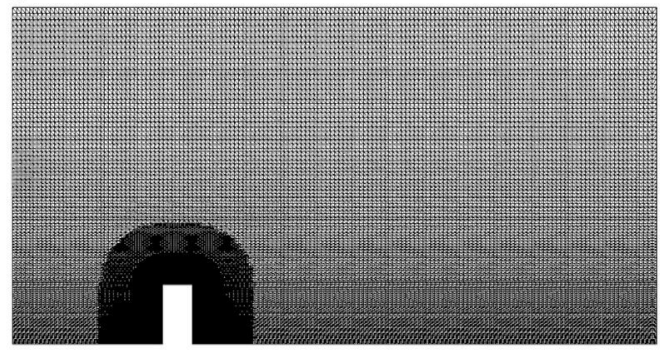

C

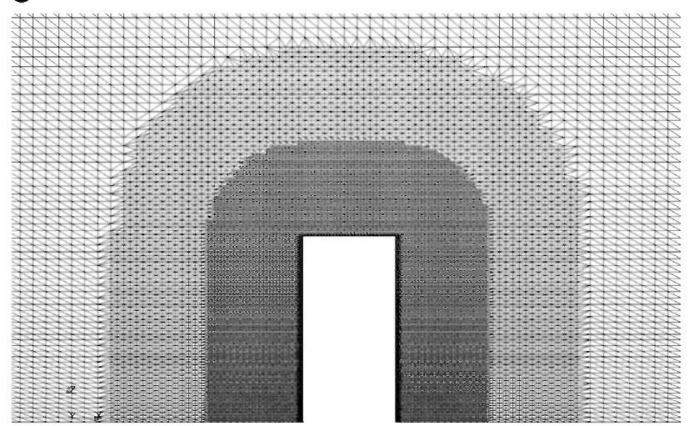

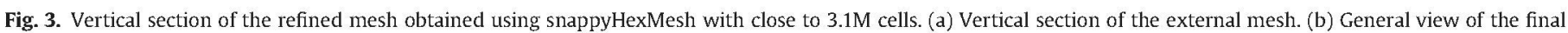
mesh. (c) Detail of the final mesh.

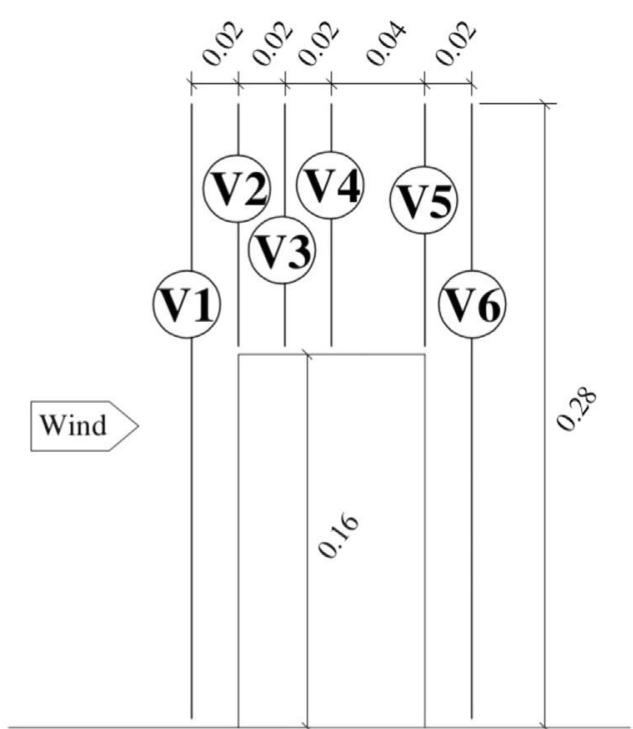

Fig. 4. Diagram of the axes (V1-V6) at the vertical section of the central part of the domain, for the comparison of the results. All lengths are in meters.

discussion about the recirculation downstream of the building. Hence, the comparison of the mean wind velocity $(U)$ and turbulent kinetic energy $(k)$ with the experimental results obtained by Meng and Hibi (1998) is carried out at the vertical axes located at the central plane of the domain according to the diagram shown in Fig. 4.

We test the RANS turbulence models shown in Table 2. Different model coefficients values are also tested (Table 3 ) for the linear $k-\varepsilon$ models (except RNG, that uses the analytically determined values for the model constants reported in the literature, Yakhot and Smith, 1992). The standard coefficients used for industrial flows are compared with those proposed by Crespo et al. (1985) and by Bechmann and Sørensen (2010). The coefficients that differ from the standard ones are $C_{\mu}, C_{\varepsilon 1}$ and $\kappa$. Crespo et al. (1985) tailored the standard model constants with the atmospheric measurements of Panofsky
Table 2

RANS turbulence models tested.

\begin{tabular}{ll}
\hline Turbulence model & Variant \\
\hline Linear $k-\varepsilon$ & Standard (SKE) \\
Linear $k-\varepsilon$ & Durbin (1996) \\
Linear $k-\varepsilon$ & Durbin-Tominaga (Tominaga et al., 2008) \\
Linear $k-\varepsilon$ & Durbin-New \\
Linear $k-\varepsilon$ & Murakami-Mochida-Kondo (MMK) (Tsuchiya et al., 1997) \\
Linear $k-\varepsilon$ & Re-normalisation group (RNG) (Yakhot and Smith, 1992; \\
& Kim and Baik, 2004) \\
Linear $k-\varepsilon$ & Yap (1987) \\
Nonlinear $k-\varepsilon$ & Shih et al. (1993) \\
$k-\omega$ SST & Shear stress transport (SST) (Menter, 1994) \\
\hline
\end{tabular}

Table 3

Tested coefficients of the linear $k-\varepsilon$ models.

\begin{tabular}{lllllll}
\hline Source coefficients & $C_{k}$ & $C_{\varepsilon 1}$ & $C_{\varepsilon 2}$ & ok & \multirow{2}{*}{$\kappa$} & $\kappa$ \\
\hline Std. coefficients & 0.09 & 1.44 & 1.92 & 1.0 & 1.3 & 0.40 \\
Crespo et al. (1985) & 0.0333 & 1.176 & 1.92 & 1.0 & 1.3 & 0.42 \\
Bechmann and Sørensen (2010) & 0.03 & 1.21 & 1.92 & 1.0 & 1.3 & 0.40 \\
\hline
\end{tabular}

and Dutton (1984). Bechmann and Sørensen (2010) use $C_{\mu}=0.03$, the appropriate value for atmospheric flows, and the standard value $\kappa=0.40$. The constant $C_{\varepsilon 1}$ is calculated using the expression

$$
C_{\varepsilon 1}=C_{\varepsilon 2}-\frac{\kappa^{2}}{\sqrt{C_{\mu}} \sigma},
$$

which is derived assuming balance between viscous dissipation and shear production in the surface layer (Bechmann and Sørensen, 2010).

The analysis of the results can focus on either qualitative or quantitative aspects. The qualitative aspects focus on the behaviour of the wind flow on the roof, and the quantitative aspects focus on the accuracy of the data compared with the experimental values. It is important to mention this because some models have very good quantitative results although they cannot reproduce 
well the recirculation on the building roof. This is because of experimental data in the size of recirculation vortex is not available (the lower points at the vertical profiles V2-V4 of Fig. 4 are above the recirculation). The opposite situation also takes place. For example, the SKE model using the coefficients proposed by Bechmann and Sørensen (2010) matches the same recirculation distance found by the experiment, but the model does not pass the validation for $k$.

Before presenting the qualitative results for all the models tested, it is appropriate to explain how we have modified the Durbin model. The Durbin model is based on the imposition of the "Realisability" constraint $2 k \geq\left\langle u_{\alpha}^{\prime} u_{\alpha}^{\prime}\right\rangle \geq 0$ via a bound on the time scale $T$ (where summation is not taken in $\left\langle u_{\alpha}^{\prime} u_{\alpha}^{\prime}\right\rangle$ ). The stagnation anomaly with impinging flows in the SKE model is addressed by relating the eddy viscosity it $_{t}$ to the turbulence velocity scale $\left\langle v_{2}^{\prime}\right\rangle$ and its time scale $T$. This procedure leads to a reduction of the $k$ overestimation at the impingement wall and to the reproduction of the recirculation flow on the roof. Although this overestimation is significatively reduced, it still remains. Another feature is that this model tends to overestimate the recirculation length both on the roof and beyond the building (Durbin, 1996). According to Durbin (1996), the factors appearing in Eq. (14) are the maximum in order to strictly apply the Realisability constraint and they can be modified to obtain agreement with experimental data, but he clearly states that this issue is beyond the scope of his article. In the present investigation, using the coefficients proposed by Crespo et al. (1985), we have empirically found that the recirculation length $X_{R}$ can be exactly matched by relaxing the Realisability constraint proposed by Durbin (1996). Note that other authors, as Tominaga et al. (2008), have also relaxed the Realisability constraint (see Eq. (15)). The coefficients of Crespo et al. (1985) are used because the hit rates are higher than those obtained using the standard coefficients with each version of the Durbin model. Additionally, the recirculation distance $X_{R}$ is nearer the experimental value than using the coefficients proposed by Bechmann and Sørensen (2010), as is shown in Table 4. In order to determine the optimum constant factor for the calculation of $T_{D}$ we have carried out a sensibility analysis of $X_{R}$ obtaining Eq. (16) as the optimum alternative. Fig. 5 shows the values tested and the corresponding result for $X_{R}$. Note that the relationship between the

Table 4

Comparison of the results using different RANS models: Reattachment distance relative to the roof length $\left(X_{R}\right)$ of the recirculation vortex on the building roof and hit rate $(H R)$ for the variables $U$ and $k$.

\begin{tabular}{lllll}
\hline RANS model & Coefficients & $X_{R}$ & $H R(\%)$ & $H R$ \\
\hline SKE & & & \\
SKE & Standard & 0.16 & 87.5 & 43.8 \\
SKE & Crespo & 0.36 & 87.5 & 56.3 \\
RNG & Bechmann & 0.52 & 87.5 & 62.5 \\
MMK & Standard & 0.62 & 87.5 & 68.8 \\
MMK & Crespo & 0.61 & 87.5 & 93.8 \\
Durbin & Bechmann & 0.84 & 87.5 & 93.8 \\
Durbin & Standard & 0.43 & 87.5 & 75.0 \\
Durbin & Crespo & 0.72 & 93.8 & 87.5 \\
Durbin-Tominaga & Bechmann & 0.86 & 87.5 & 87.5 \\
Durbin-Tominaga & Standard & 0.29 & 87.5 & 56.3 \\
Durbin-Tominaga & Crespo & 0.60 & 87.5 & 75.0 \\
Durbin-New & Bechmann & 0.74 & 87.5 & 75.0 \\
Yap & Crespo & 0.52 & 87.5 & 75.0 \\
Yap & Standard & 0.40 & 87.5 & 43.8 \\
Nonlinear Shih & Bechmann & 0.74 & 81.3 & 75.0 \\
$k-\omega$ SST & Standard & $>1$ & 87.5 & 68.8 \\
Experimental (Meng and Hibi, 1998) & Standard & $>1$ & 87.5 & 43.8 \\
\hline
\end{tabular}

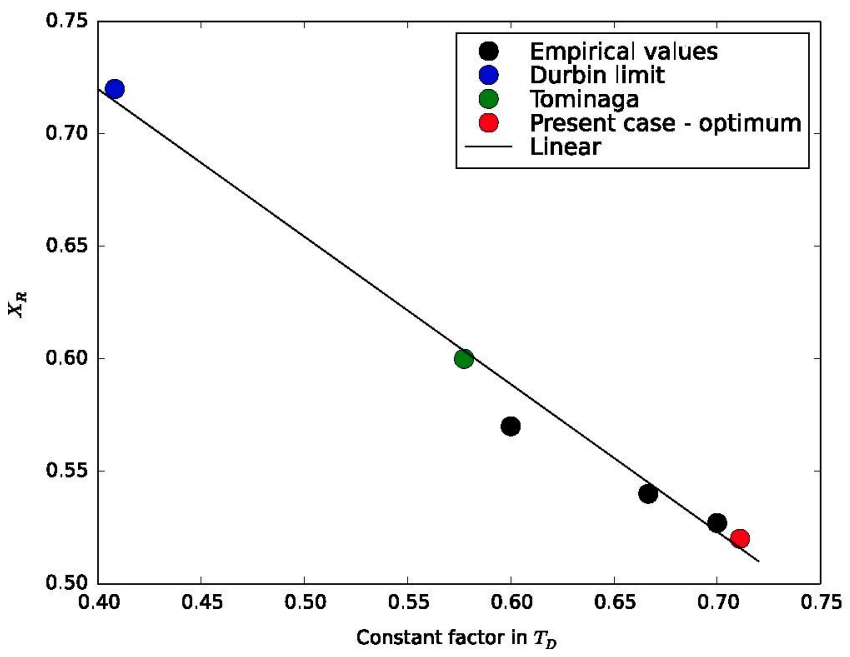

Fig. 5. Sensitivity analysis for the recirculation distance $X_{R}$ by varying the constant factor in the definition of $T_{D}$ (Eq. (16)).

constant factors in $T_{D}$ expression (16) and $X_{R}$ is linear.

The most important aspects for the qualitative analysis are the wind direction and the recirculation distance. The wind direction at each region of the building roof is analysed in Section 4.2. Regarding the recirculation of the flow, the comparison of the simulation results obtained for the reattachment distance $\left(X_{R}\right)$, relative to the roof length, of the recirculation vortex on the building roof is presented in Table 4 . As is well known, the SKE model cannot predict well the reverse flow on the building roof due to its overprediction of the turbulent kinetic energy at the impingement region of the windward wall (Shao et al., 2012b) and, because of that, the use of SKE is not recommended to estimate the wind behaviour in the urban environment (Franke et al., 2007; Guidebook for Practical Applications of CFD to Pedestrian Wind Environment around Buildings, 2013). Using the SKE with the coefficients of Bechmann and Sørensen (2010), the same value of $X_{R}$ from the experiment of Meng and Hibi (1998) is obtained, although the case is not successfully validated for $k$. The Durbin model used by Tominaga et al. (2008) with the coefficients proposed by Crespo et al. (1985) overestimates $X_{R}$ by only $15.4 \%$, giving the closest agreement with the experimental data among the different models tested. Additionally, the MMK model using the coefficients of Crespo et al. (1985) overestimates $X_{R}$ by a $17.3 \%$ and the original Durbin with standard coeffs. underestimates it by a $17.3 \%$. The Durbin model with the $T_{D}$ proposed in Eq. (16) shows the best agreement using the coefficients of Crespo et al. (1985), reaching the same value than the experiment of Meng and Hibi (1998), $X_{R}=0.52$. Therefore, these models are the most appropriate to estimate the behaviour of the wind flow over a building roof with a qualitative approach. Both Nonlinear Shih and $k-\omega$ SST models overestimate the recirculation beyond the roof. All the $k-\varepsilon$ models tested present a better agreement with the experimental data of Meng and Hibi (1998) by using the coefficients proposed by Crespo et al. (1985) and by Bechmann and Sørensen (2010) than by using the standard coefficients.

Table 4 also shows the hit rates $(H R)$ for streamwise velocity $(U)$ and the turbulent kinetic energy $(k)$. These hit rates, evaluated at the vertical axes V2-V5 of Fig. 4 (building roof), are calculated according to Santiago et al. (2007) by using the equation

$H R=\frac{1}{n} \sum_{i=1}^{n} N_{i}$ 
where $n$ is the total number of points compared, and

$N_{i}=1$ if $\left|\frac{S I M_{i}-E X P_{i}}{E X P_{i}}\right| \leq R D$ or $\left|S I M_{i}-E X P_{i}\right| \leq A D$

$N_{i}=0$ else,

where $S I M_{i}$ and $E X P_{i}$ are the simulation and experimental values, and $R D$ and $A D$ are the relative and absolute maximum admissible deviation from the experimental data, respectively. These values are $R D=0.25$ and $A D=0.05$ and $A D=0.017$ for $U$ and $k$, respectively (Santiago et al., 2007). The values of the hit rate that allow us to consider the validation work as successful are $H R \geq 66 \%$.

In this case, all the models reach streamwise velocity hit rates higher than $80 \%$, which is a reasonably good result. The most important difficulty for the RANS models is to estimate the turbulent kinetic energy. In this case, the only models that reach hit a

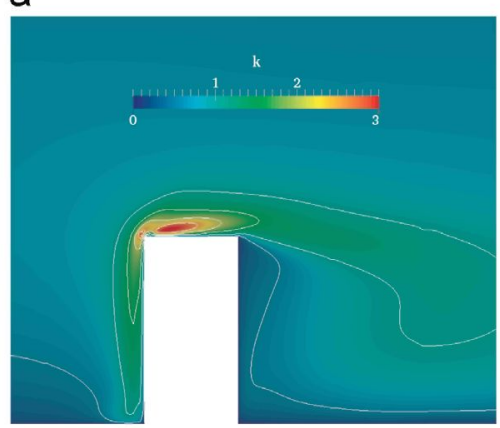

d

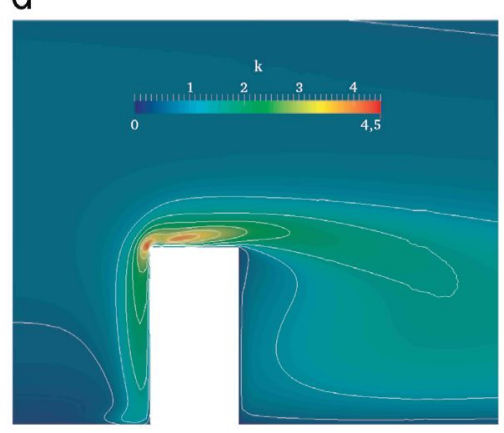

g

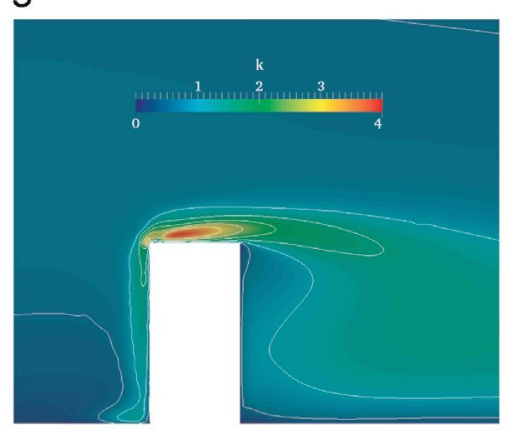

j

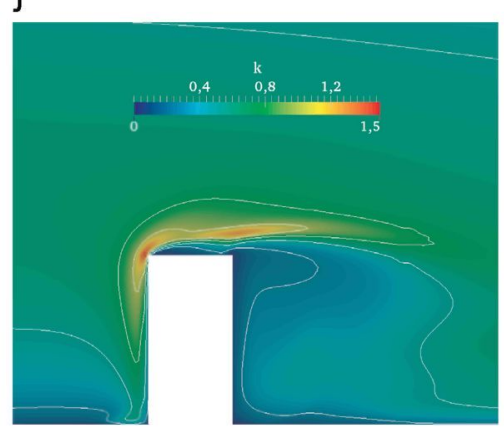

b

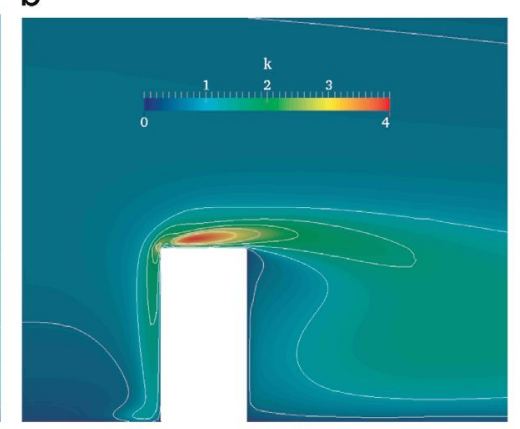

e

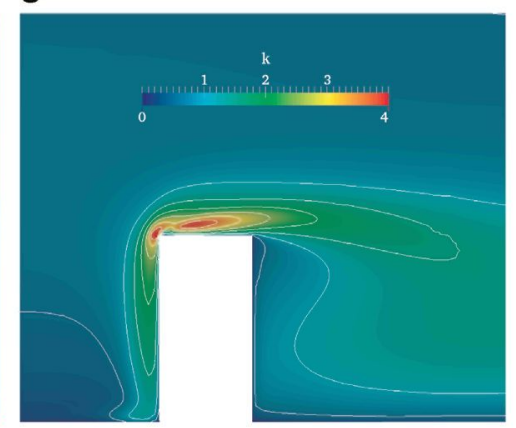

h

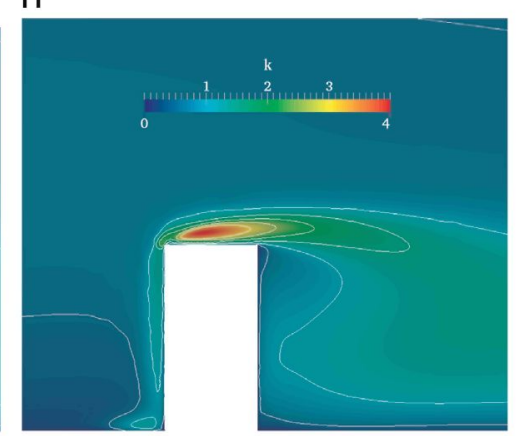

k

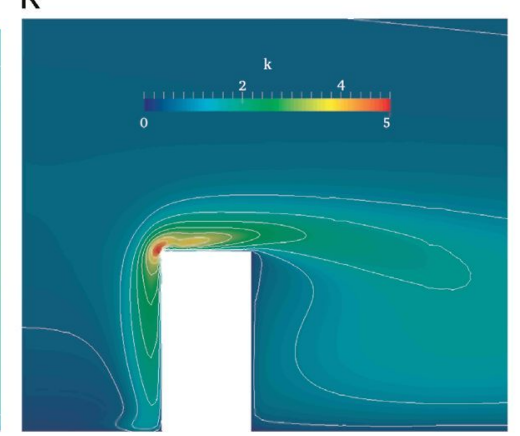

C

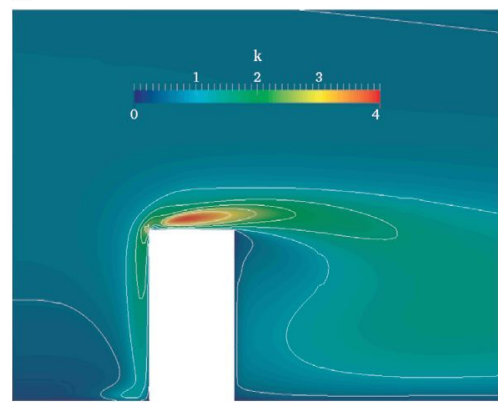

f

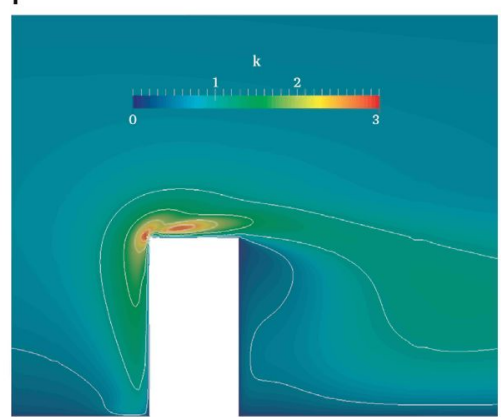

i

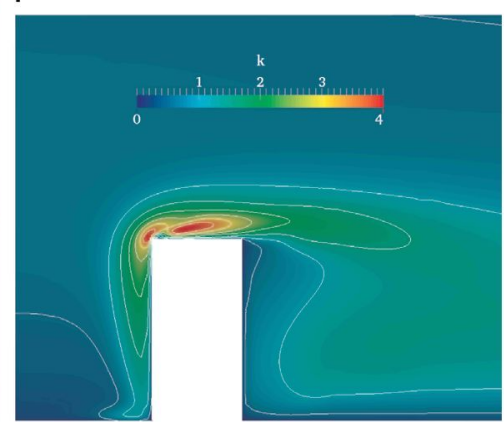

I

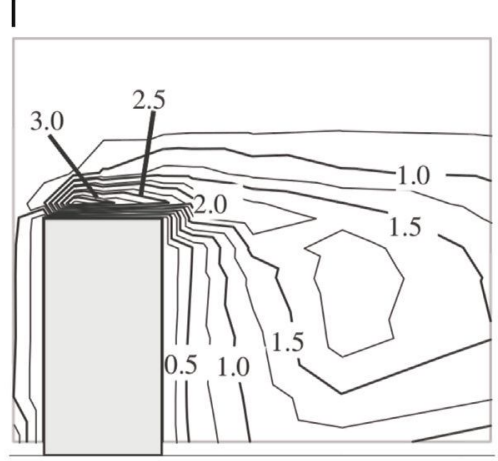

Fig. 6. Comparison of the turbulent kinetic energy $\left(\mathrm{m}^{2} / \mathrm{s}^{2}\right)$ at the vertical section at the centre of the domain, using the models with $H R \geq 66 \%$. (a) Durbin-Standard. (b) Durbin-Crespo. (c) Durbin-Bechmann. (d) Durbin-Tominaga-Crespo. (e) Durbin-Tominaga-Bechmann. (f) RNG-Standard. (g) MMK-Crespo. (h) MMK-Bechmann. (i) YapBechmann. (j) Nonlinear Shih-Standard. (k) Durbin-New-Crespo. (1) Exp. (Tominaga et al., 2008). (For interpretation of the references to colour in this figure caption, the reader is referred to the web version of this paper.) 
rates above $66 \%$ are the RNG (with standard coeffs.), MMK (with Crespo and Bechmann coeffs.), all the Durbin variations (with Crespo and Bechmann coeffs., and the original Durbin model also with standard coeffs.), Yap (with Bechmann coeffs.) and Nonlinear Shih.

The most important aspects of the quantitative analysis are the accuracy of the streamwise velocity and turbulent kinetic energy. All the models tested successfully passed the validation for $U$ and, therefore, the critical point for the RANS models is to accurately predict the turbulent kinetic energy. Fig. 6 shows a comparison of the turbulent kinetic energy for the models that successfully pass the validation for $k$. The highest values of $k$ at the impingement region of the windward wall (shown in red), are always overestimated at the upstream corner. However, these highest values are also found on the central region of the building roof, in better agreement with the experimental results. The results using the MMK model (with Bechmann coeffs.) present the best agreement with the experimental data, showing peak values of $k$ only on the central part of the roof. The MMK model avoids the overestimation of the production term of $k\left(R_{k}\right)$ by using both strain rate scale $S$ and vorticity scale $\Omega$ (instead of only $S$, as in the SKE model) in the calculation of $P_{k}$ when $\Omega<S$. The original Durbin models (with Crespo and Bechmann coeffs.) and the MMK-Crespo also show a qualitatively good result, since the peak values around the upstream edge have a lower magnitude than on the central region. All the models that successfully pass the validation for $k$ are exhaustively analysed in Section 4.2, focussing on the accuracy of the different models at the different regions of the building roof.

\subsection{Grid dependency study}

In order to check the order of convergence of the solutions, the new variation of the Durbin model proposed in this investigation (the best results found) is analysed using 3 different meshes: coarse, medium-size (the size used for the comparison of the models) and fine mesh. The refinement distances around the building surfaces are controlled by the snappyHexMesh application of OpenFOAM. Table 5 presents the main parameters used, and Fig. 7 shows a detail of the final meshes obtained. Table 5 also shows the hit rates obtained at the 3 cases. The hit rate obtained for $k$ shows a variation between the 3 cases.

Table 5

Main parameters of the mesh refinement using the snappyHexMesh application of OpenFOAM, and values obtained for the hit rates $(H R)$.

\begin{tabular}{llll}
\hline Parameter & Coarse mesh & Medium-size mesh & Fine mesh \\
\hline Refinement distance & 0.01 & 0.16 & 0.32 \\
Total number of cells & $1.7 \mathrm{M}$ & $3.1 \mathrm{M}$ & $9.8 \mathrm{M}$ \\
$H R(\%)$ & 87.5 & 87.5 & 87.5 \\
$H R_{k}(\%)$ & 68.8 & 75.0 & 81.3 \\
\hline
\end{tabular}

To check the order of convergence of the numerical scheme, the grid convergence index $(G C I)$ is calculated according to Roache (1998) by using the total number of cells $(N)$ and the hit rate of $k$ $\left(H R_{k}\right)$. Since we use 3 different meshes, the grid convergence index is

$G C I=1.25 \frac{\left|e_{21}\right|}{r_{21}^{p}-1}$,

where the relative error is

$e_{21}=\frac{H R_{k, 2}-H R_{k, 1}}{H R_{k, 1}}$

the effective grid refinement ratio for a 3-dimensional unstructured grid is

$r_{21}=\left(\frac{N_{1}}{N_{2}}\right)^{1 / 3}$,

and the observed convergence rate is

$p=\frac{|\ln | \frac{H R_{k, 3}-H R_{k, 2}}{H R k, 2-H k_{k, 1}}|+q(p)|}{\ln r_{21}}$,

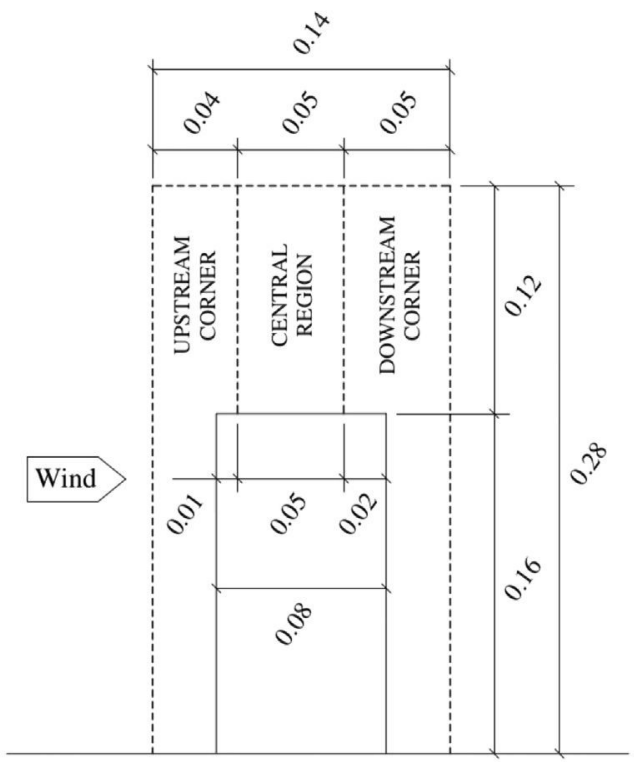

Fig. 8. Diagram of the regions of the building roof for the flow analysis. All lengths are in meters a

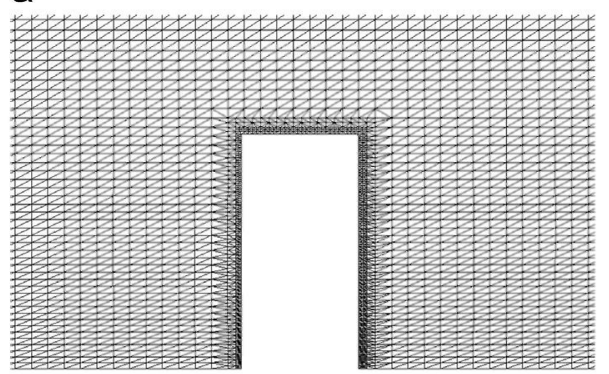

b

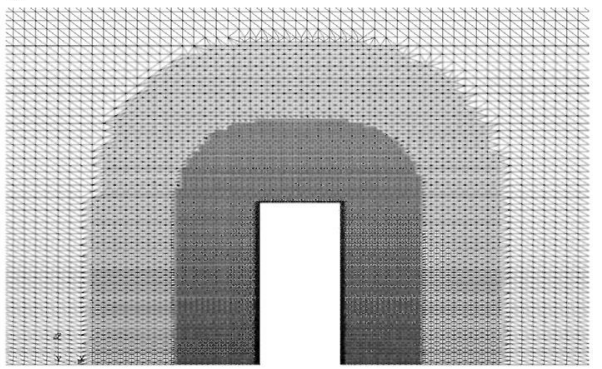

c

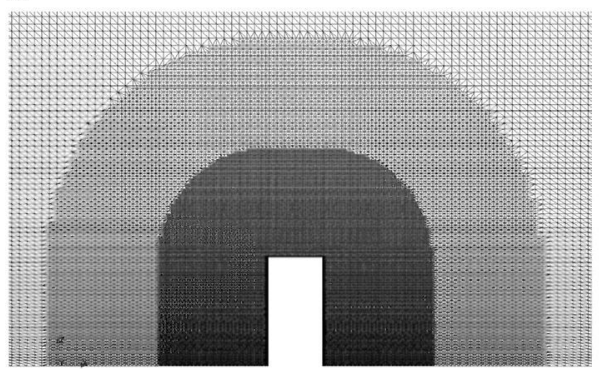

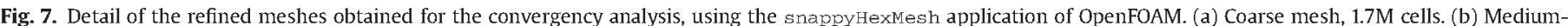
size mesh, 3.1M cells. (c) Fine mesh, 9.8M cells. 

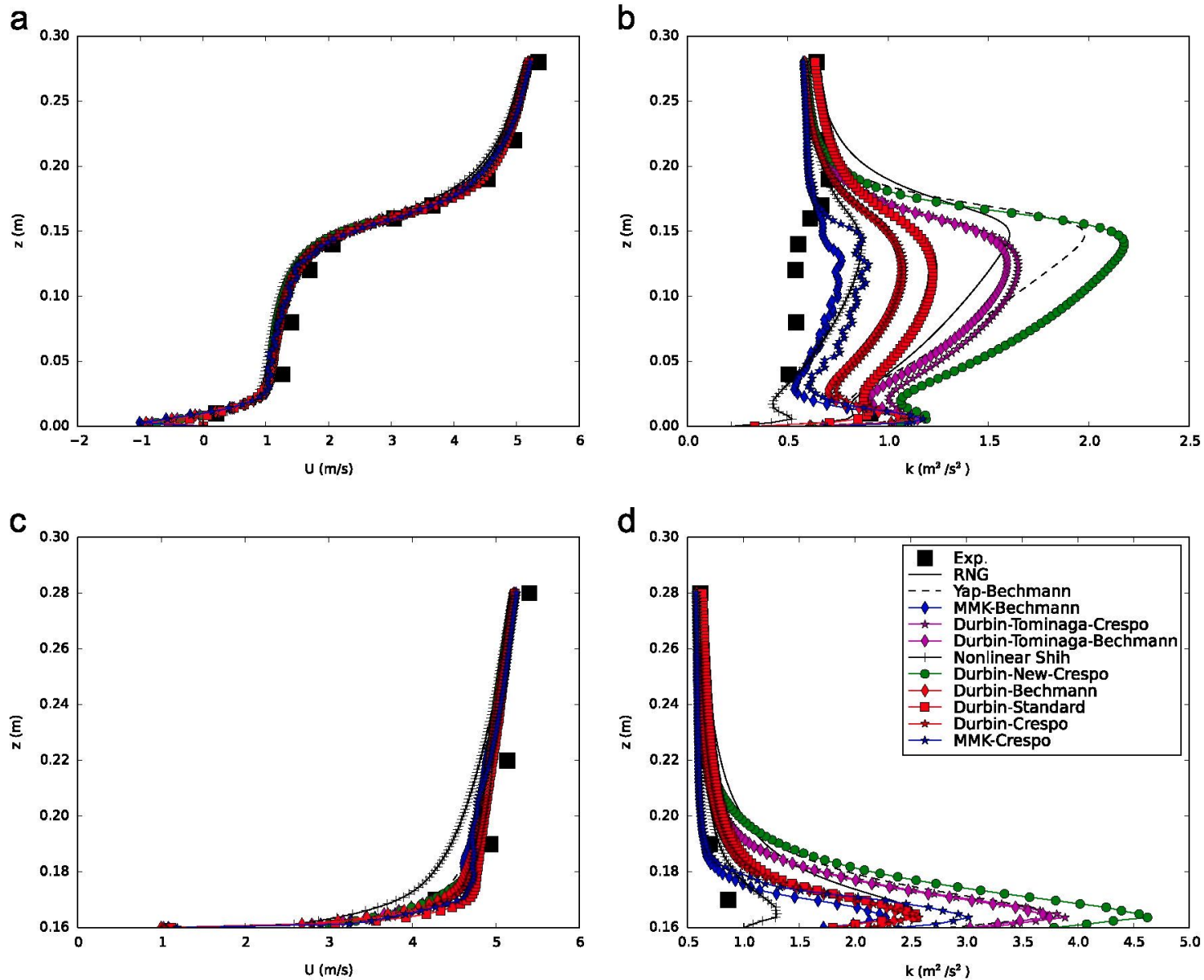

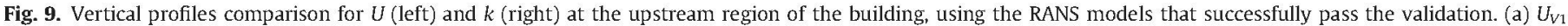
(b) $k_{V 1}$. (c) $U_{V 2}$. (d) $k_{V 2}$

where

$q(p)=\ln \left(\frac{r_{21}^{p}-s}{r_{32}^{p}-s}\right)$

where

$s=\operatorname{sgn}\left(\frac{H R_{k, 3}-H R_{k, 2}}{H R_{k, 2}-H R_{k, 1}}\right)$.

Note that the system of Eqs. (29) and (30) must be solved by means of an iterative procedure, starting with $q=1$. The subscripts 1,2 and 3 above refer to the fine, medium-size and coarse meshes, respectively. In this case, the observed convergence rate is $p=2.23$ and the grid convergence index is $G C_{21}=0.0577$ (5.77\%).

\subsection{Flow behaviour on different regions of the building roof}

Since the different models analysed show different agreement with the experimental data in the different regions of the building roof, it is convenient to divide the roof into different regions according to the characteristics of the flow, in order to recommend the most appropriate model to deal with each specific problem. Therefore, the building roof is divided into 3 regions: upstream corner, central region and downstream corner of the roof (Fig. 8). The upstream corner of the roof corresponds to the impingement region, the central region corresponds to the recirculation area and the downstream corner corresponds to the leaving flow region. In what follows, the most appropriate models to estimate the velocity and turbulent kinetic energy at each region are identified.

\subsubsection{Upstream corner of the roof}

Fig. 9a and $c$ shows the velocity profiles obtained around the upstream corner of the building roof (vertical axes shown in Fig. 4), using the RANS models that successfully pass the validation, compared with the experimental data of Meng and Hibi (1998). The agreement of the velocity profiles with the experimental results is reasonably good. The worst option to estimate the velocity upstream is the Nonlinear model of Shih et al. (1993).

Fig. $9 \mathrm{~b}$ and $\mathrm{d}$ shows the turbulent kinetic energy profiles obtained around the upstream corner of the building roof (vertical axes shown in Fig. 4), using the RANS models that successfully pass the validation, compared with the experimental data of Meng and Hibi (1998). The best models to estimate the turbulent kinetic energy upstream are the MMK and Nonlinear Shih. At the upstream edge, only the Nonlinear Shih model shows a reasonably good result. The rest of the models clearly overestimate $k$ at $z<0.22 \mathrm{~m}$. Due to the relaxation of the Realisability constraint, the modified Durbin model proposed in the present investigation shows the highest overestimation at the impingement region, although it shows an excellent agreement with the experimental data at the centre and downstream regions.

For the estimation of both $U$ and $k$ upstream, the best RANS model is the MMK, obtaining hit rates of $H R_{U}=92.9 \%$ and $H R_{k}=71.4 \%$ at this region for $U$ and $k$, respectively.

\subsubsection{Central region of the roof}

Fig. 10a and c shows the velocity profiles obtained at the central region of the building roof (vertical axes shown in Fig. 4), using the RANS models that successfully pass the validation, compared with 
a

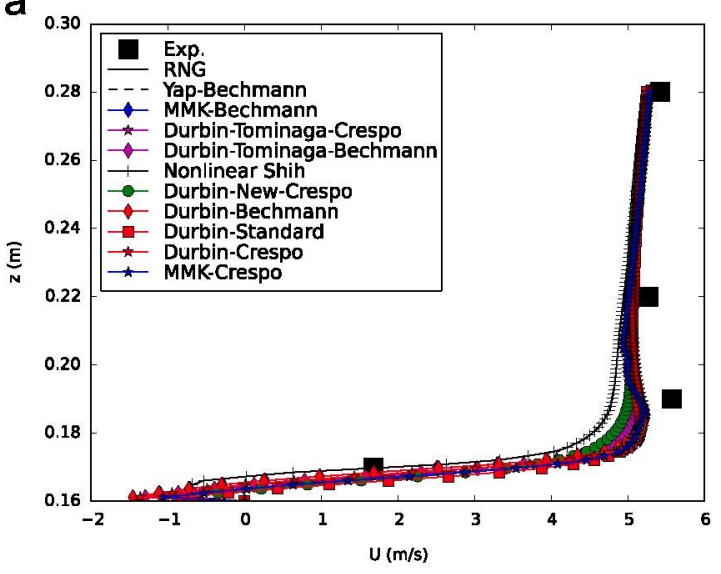

C

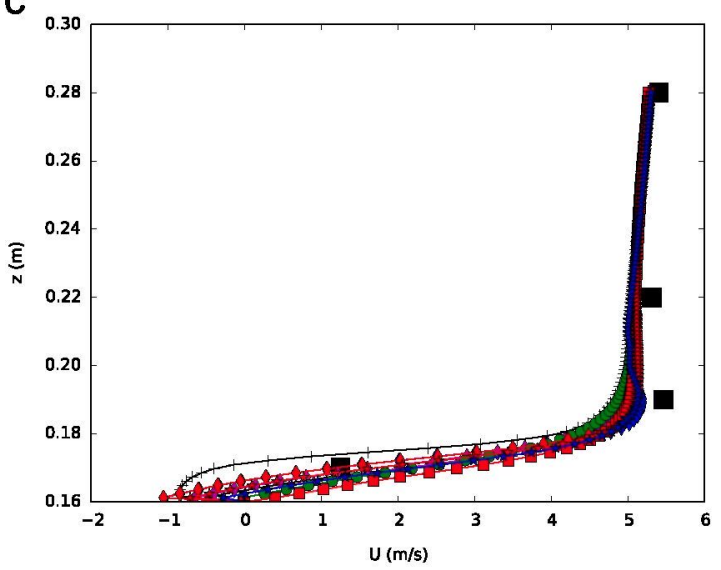

b

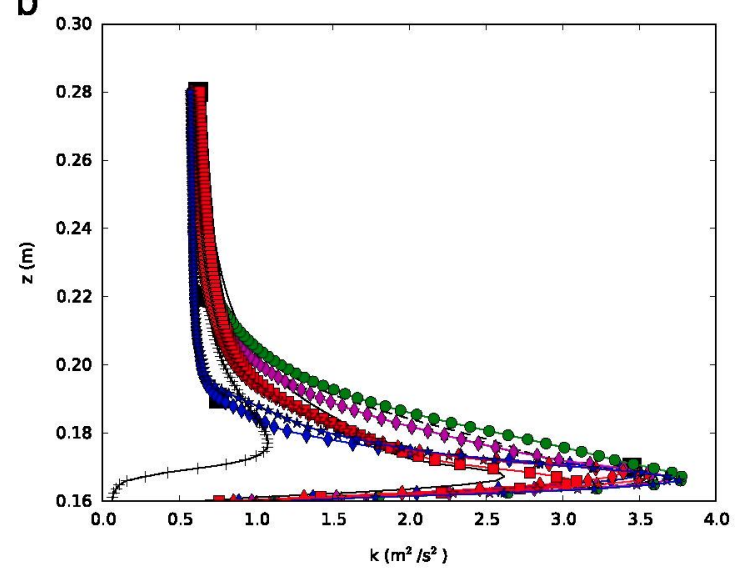

d

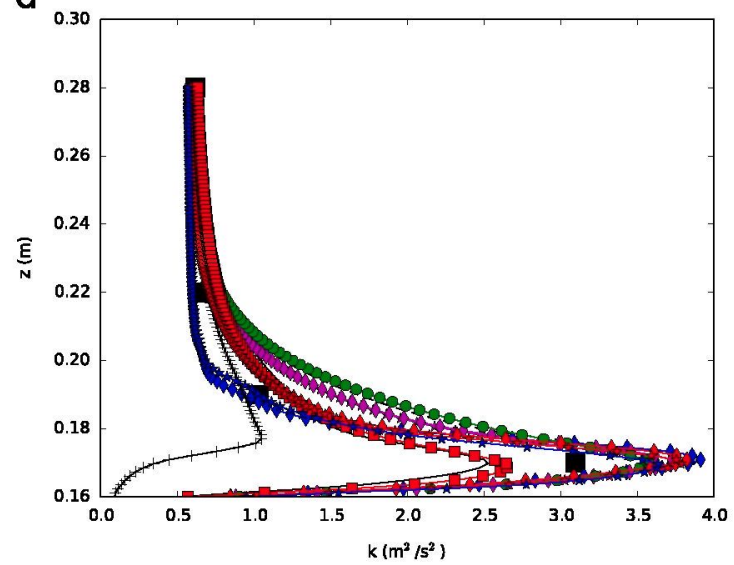

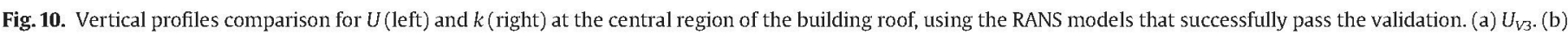
$k_{V 3}$. (c) $U_{V 4 \cdot}$ (d) $k_{V / 4}$

the experimental data of Meng and Hibi (1998). All the models show a reasonably good agreement with the experimental data, reaching hit rates of $H R_{U}=75-87.5 \%$. The Nonlinear Shih model shows the worst behaviour. However, there is an uncertainty regarding the velocity in the recirculation because the experimental results are only presented from the top of the vortex, and the maximum velocity below (negative horizontal component) is not provided by the experimental results. The reattachment distance $\left(X_{R}\right)$ is the only variable available to analyse the qualitative behaviour of the recirculation flow. From the qualitative point of view, the best agreement is obtained with the new modification proposed for the Durbin model, yielding exactly the same value of the reattachment distance obtained at the experiment of Meng and Hibi (1998). It is important to mention that the Nonlinear Shih model clearly overestimates the reattachment distance.

Fig. 10b and d shows the turbulent kinetic energy profiles obtained at the central region of the building roof (vertical axes shown in Fig. 4), using the RANS models that successfully pass the validation, compared with the experimental data of Meng and Hibi (1998). The Nonlinear shih model clearly underestimates $k$ at $z<0.18 \mathrm{~m}$ up to $60 \%$. The rest of the models show a reasonably good agreement with the experimental results. At the lower height of the region $(z<0.18 m)$ RNG and the original Durbin (with standard coeffs.) models underestimate $k$ by $15 \%$. The rest of the models overestimate $k$ by a similar amount. The best model to estimate $k$ at the central region of the roof is the MMK, that reaches a $H R_{k}=100 \%$.

\subsubsection{Downstream corner of the roof}

Fig. 11a and $\mathrm{c}$ shows the velocity profiles obtained around the downstream corner of the building roof (vertical axes shown in Fig. 4), using the RANS models that successfully pass the validation, compared with the experimental data of Meng and Hibi (1998). The agreement with the experimental results is reasonably good for all the models. The worse behaviour is observed for the Nonlinear Shih profiles, where $U$ is notably underestimated due to the recirculation overestimation.

Fig. $11 \mathrm{~b}$ and $\mathrm{d}$ shows the turbulent kinetic energy profiles obtained around the downstream corner of the building roof (vertical axes shown in Fig. 4), using the RANS models that successfully pass the validation, compared with the experimental data of Meng and Hibi (1998). Nonlinear Shih, RNG and the original Durbin (with standard coeffs.) models clearly underestimate $k$. The rest of the models show a reasonably good agreement with the experimental data, especially MMK (with Crespo coeffs.), the new modified version of Durbin (with Crespo coeffs.) and Yap (with Bechmann coeffs.). The exception is downstream of the building (close to the vertical wall of the building), where RNG and the original Durbin (with standard coeffs.) present the best agreement.

\subsection{Wind energy exploitation and wind turbine positioning}

Defining the concentration factor of the wind as the increase of the velocity around the building compared to the freestream inlet velocity at the height of the building (Lu and Ip, 2009), a concentration factor of the wind of $6-20 \%$ on the building roof is appreciated at the vertical axes V2-V5. However, atmospheric 

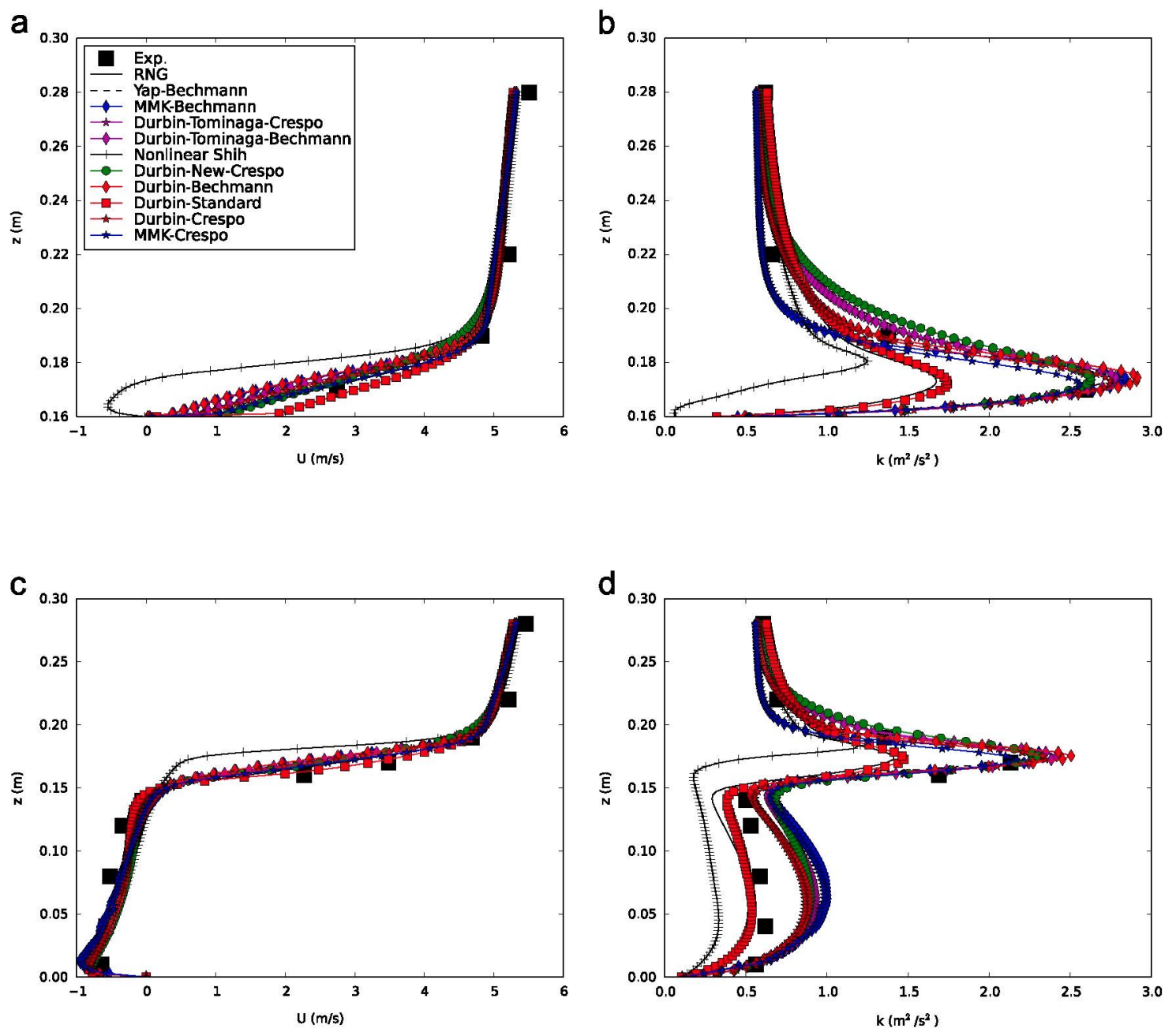

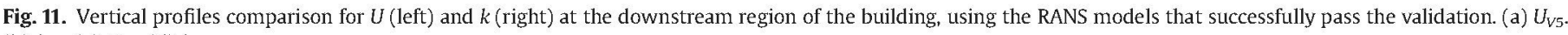
(b) $k_{V 5}$. (c) $U_{V 6}$. (d) $k_{V 6}$.

wind turbulence is one of the main effects causing fatigue damage on wind turbine components (Mouzakis et al., 1999). According to the European Wind Turbine Standards II (Pierik et al., 1999), when the turbulence intensity exceeds 15\% the fatigue loads on the wind turbines have to be re-evaluated based on the actual conditions at the site. Therefore, a value of $\Pi=0.15$ is used as a maximum admissible for the horizontal axis wind turbines (HAWT). Fig. 12a shows a diagram with the most adequate type of wind turbine for each region, plotted on top of the turbulence intensity map and the velocity field. The bold line represents the limit between the most appropriate area for HAWT and vertical axis wind turbines (VAWT). The most appropriate areas to install HAWT are above $z / H=0.19$ from the roof surface upstream and above $z / H=0.31$ downstream. It is recommended to incline the horizontal axis of the HAWT $5^{\circ}$ downwards at the upstream region below $z / H=0.31$. Below these heights, the installation of a VAWT is more appropriate since it is not affected by the wind direction fluctuations (Kooiman and Tullis, 2010) and it resists better the velocity fluctuations of the wind (Carpman, 2011). Additionally, the VAWT can be installed in horizontal position at the central-upstream region close to the roof surface, in order to take the most of the recirculation of the flow. That is, the VAWT in horizontal position can exploit the positive streamwise velocity up and the negative streamwise velocity down and, additionally, it can exploit the circular flow upstream or donwnstream of the turbine. More investigations are needed to better understand the behaviour of the VAWT in strongly turbulent environments.
Another very interesting application for the wind energy exploitation at the upstream corner of the building roof is the use of a ducted wind turbine (Toja-Silva et al., 2013). This kind of wind turbine takes the most of the pressure difference between the vertical wall and the roof, with positive and negative pressures, respectively. Depending on the differential pressure coefficient, power coefficient values close to 1 can be reached (Grant et al., 2008). Fig. 12b shows a diagram of the turbine position into the pressure field.

High wind situations (wind speed $>3 \mathrm{~m} / \mathrm{s}$ as the one studied in this paper show a well defined circulation pattern and a predominant wind direction (Whiteman, 2000; Jiménez and Dudhia, 2013). However, in order to make the conclusions of this paper more general, and independent of the incident wind direction, we also consider an oblique incident wind direction $\left(45^{\circ}\right)$. Fig. 13 shows a comparison between the threshold of $T I=0.15$ for a normal incident wind direction $\left(0^{\circ}\right)$ and an oblique one $\left(45^{\circ}\right)$. For the oblique wind direction, the HAWT can be placed above $z / H=0.14$ and $z / H=0.27$ at upstream and downstream edges, respectively. Below these heights, VAWT must be considered. Since the threshold of $\Pi I=0.15$ is lower everywhere, the heights obtained for the normal incident wind (most unfavourable case) are conservative values for a general wind direction case. Therefore, we conclude that, in general, an HAWT can be placed above $z / H=0.31$ anywhere at the roof, regardless of the incident wind direction. The situation investigated in this paper is an isolated building but, since the inlet profile is highly turbulent (as shown in 
a

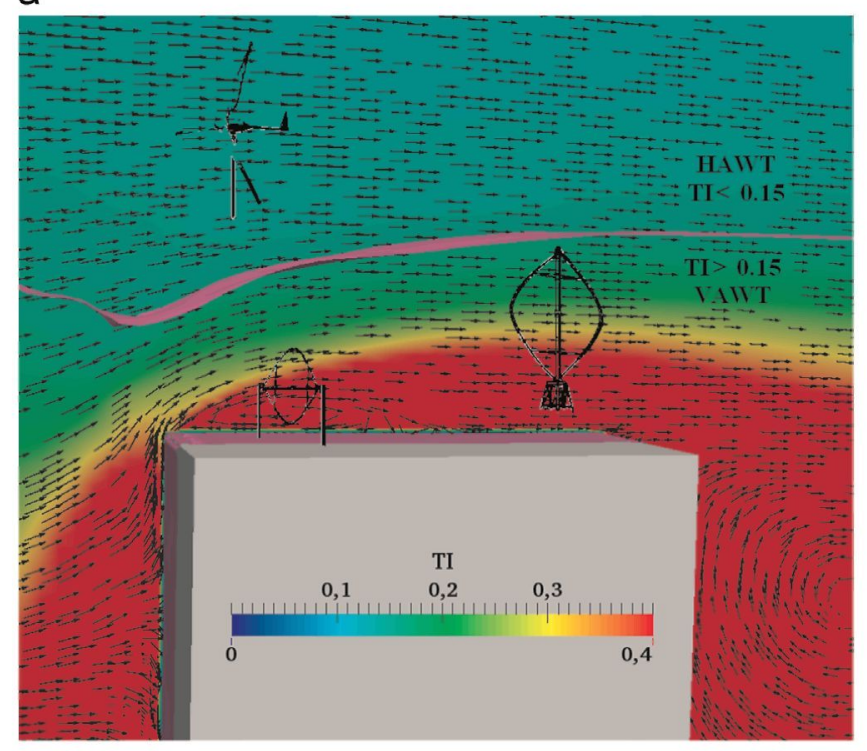

b

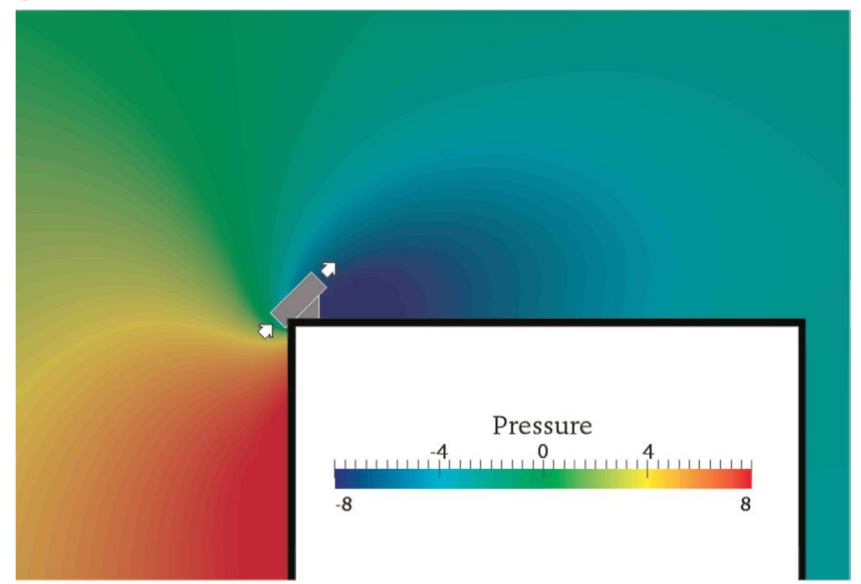

Fig. 12. Wind turbine positioning diagrams. (a) Most appropriate wind energy exploitation systems at the different regions of the building roof. The vector field is the velocity, the background colourmap is turbulence intensity $(T I)$ and the bold line (in magenta) is an isocontour of the isoline corresponding to $\pi=0.15$. (b) Ducted wind turbine at the upstream corner of the roof into the pressure field. (For interpretation of the references to colour in this figure caption, the reader is referred to the web version of this paper.)

Fig. 2b), our conclusions are also valid for a building surrounded by smaller buildings.

\section{Conclusions}

In this investigation, CFD simulations of the wind flow around a single building were performed with OpenFOAM using several RANS turbulence models, and the results were compared with the experimental results of a wind tunnel benchmark case. The behaviour of the different RANS models were analysed at different regions of the building roof (upstream, downstream and central region of the roof), and the most appropriate models were identified for each situation both qualitatively and quantitatively. Furthermore, the characteristics of the flow were studied from the point of view of the wind energy exploitation and the most adequate turbine model for each region was suggested. To the knowledge of the authors, there are not publications that suggest a procedure for HAWT positioning in high-rise building roofs.
On the qualitative side, the Durbin model with the $T_{D}$ form proposed in Eq. (16) showed a perfect agreement with the experimental data for recirculation distance. Additionally, DurbinTominaga (with Crespo coeffs.), MMK (with Crespo coeffs.) and original Durbin (with standard coeffs.) models showed a reasonable agreement with the experiment. The SKE model (with Bechmann coeffs.) also matched the same value of the recirculation distance obtained by the experiment, but it is not successfully validated for $k$. Both Nonlinear Shih and $k-\omega$ SST models overestimate the recirculation beyond the roof. All the $k-\varepsilon$ models tested show a better agreement with the experimental data by using the coefficients proposed by Crespo et al. (1985) and by Bechmann and Sørensen (2010) than by using the standard coefficients.

For the quantitative analysis, the hit rate is calculated. All the models successfully passed the validation threshold $(H R>66 \%$ ) for the streamwise velocity $(U)$ but, for the turbulent kinetic energy ( $k$ ) only the RNG (with standard coeffs.), MMK (with Crespo and Bechmann coeffs.), all the Durbin variations (with Crespo and Bechmann coeffs., and the original Durbin model also with standard coeffs.), Yap (with Bechmann coeffs.) and Nonlinear Shih passed the validation.

In order to check the order of convergence of the solutions, the hit rate for $k$ obtained with the new Durbin $T_{D}$ proposed in this investigation is analysed for 3 different meshes: coarse, mediumsize and fine mesh $(1.7 \mathrm{M}, 3.1 \mathrm{M}$ and $9.8 \mathrm{M}$ cells, respectively). The observed convergence rate is $p=2.23$ and the grid convergence index is $G C I=0.0577$ ( $5.77 \%$ ).

Regarding the behaviour of the RANS models at the different regions of the building roof, at the upstream region of the roof the best agreement with experimental data for both $U$ and $k$ is achieved using the MMK model (with both Crespo and Bechmann coeffs.). At the central region of the roof there is an uncertainly regarding the velocity in the recirculation zone because the experimental data do not include the negative horizontal component of velocity. The reattachment distance $\left(X_{R}\right)$ is the only data available to analyse the qualitative behaviour of the recirculation. From this qualitative point of view, the best agreement is obtained with the new modification of the Durbin model (with Crespo coeffs.), yielding exactly the same value than the experimental one. At the central region of the roof the best agreement for $k$ is obtained with the MMK model (with both Crespo and Bechmann coeffs.) that reach a $H R_{k}=100 \%$, although the rest of the linear turbulence models show a reasonably good agreement. At the downstream region of the building roof (on the downstream edge), MMK (with Crespo coeffs.), the new modified version of Durbin (with Crespo coeffs.) and Yap (with Bechmann coeffs.) shows the best agreement for both $U$ and $k$. The Durbin model with the proposed form of $T_{D}$ and Crespo coefficients gave the best results from a global point of view, both qualitatively and quantitatively. Additionally, the MMK model (with Crespo coeffs.) gave the best quantitative results and a reasonably good qualitative agreement with the experimental data.

The analysis of the turbines positioning is based in the turbulent kinetic energy, limited up to a turbulence intensity $T I<0.15$ for HAWT, from a fatigue loads preventing point of view. According to that, the most appropriate areas found to install HAWT are above $z / H=0.19$ from the roof surface upstream and above $z / H=0.31$ downstream. It is recommended to incline the horizontal axis of the HAWT $5^{\circ}$ downwards at the upstream region below $z / H=0.31$. The installation of VAWT is recommended below these heights. The installation of a VAWT in horizontal position at the central-upstream region close to the roof surface was also considered, to make the most of the recirculation of the flow. Additionally, the installation of a ducted wind turbine at the upstream corner of the building roof, in order to make the most of 
a

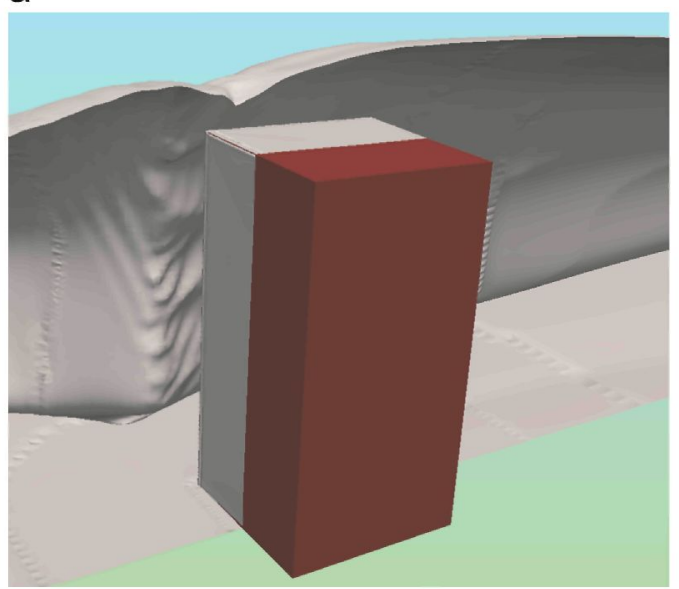

b

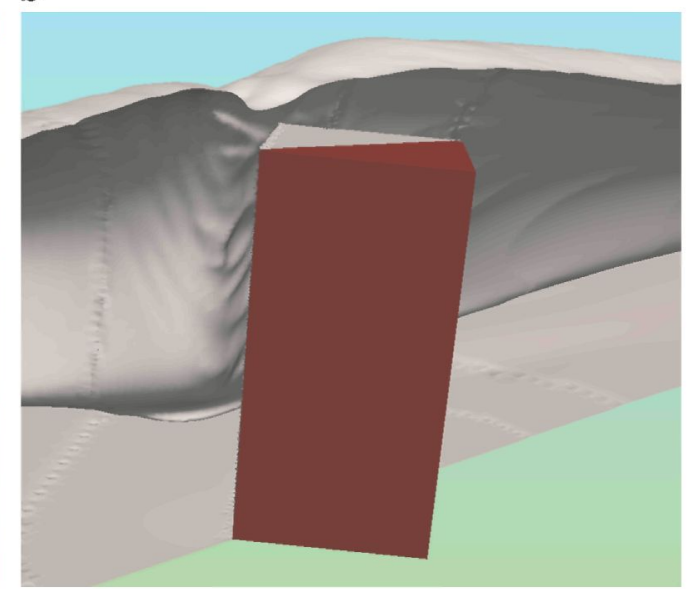

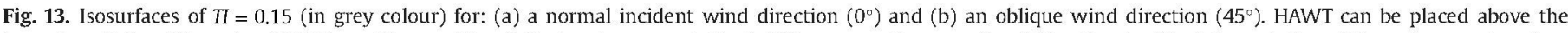

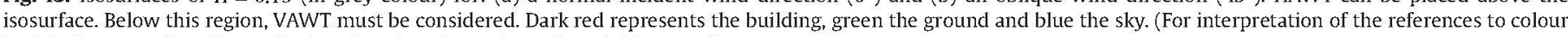
in this figure caption, the reader is referred to the web version of this paper.)

the pressure difference between the vertical wall (positive) and the roof surface (negative), is also interesting. Although high wind situations as the one studied in this paper show a predominant wind direction, in order to make the conclusions of this paper more general, and independent of the incident wind direction, we also consider an oblique incident wind direction. The results show that HAWT can be placed above $z / H=0.14, z / H=0.27$ at the upstream and downstream edges, respectively. Since the threshold of $T I=0.15$ is lower everywhere, the heights obtained for the normal incident wind (most unfavourable case) are conservative values for a general wind direction case and, therefore, can be concluded that an HAWT can be placed above $z / H=0.31$ everywhere at the investigated case, regardless of the incident wind direction. The situation investigated corresponds to an isolated building but, since the inlet profile is highly turbulent, this rule can also be applied to a building surrounded by smaller buildings.

In order to be rigorous in the extrapolation of the results to a real case, there is a need to carry out a benchmark case similar to the one analysed in this investigation but with a full-scale geometry. We only analysed the regions on the central symmetry axis of the roof, because the experimental data is only available at these positions. To complete the analysis it would be interesting to have also experimental data at the corners and other regions of interest apart from the central axis. Additionally, a more realistic urban setting with several buildings included should also be considered. The authors of this investigation are currently working on this matter.

\section{Acknowledgements}

The authors acknowledge the computer time provided by the Facility for Large-scale Computations in Wind Energy Research (FLOW) at the University of Oldenburg and the Euler cluster at the CIEMAT.

\section{References}

Abohela, I., Hamza, N., Dudek, S., 2013. Effect of roof shape, wind direction, building height and urban configuration on the energy yield and positioning of roof mounted wind turbines. Renew. Energy 50, 1106-1118.

ANSYS Fluent, 2015. Web: 〈http://aerojet.engr.ucdavis.edu/fluenthelp/html/ug/ node992.htm\}.
Bakker, A., 2015. Applied Computational Fluid Dynamics. Web: 〈http://www.bakker org/dartmouth06/engs 150/05-solv.pdf).

Balogh, M., Parente, A., Benocci, C., 2012. RANS simulation of ABL flow over complex terrains applying an Enhanced $k-\varepsilon$ model and wall function formulation: implementation and comparison for Fluent and OpenFOAM. J. Wind Eng. Ind. Aerodyn. 106, 360-368.

Bechmann, A., Sørensen, N.N., 2010. Hybrid RANS/LES method for wind flow over complex terrain. Wind Energy 13, 36-50.

Blocken, B., 2014. 50 years of computational wind engineering: past, present and future. J. Wind Eng. Ind. Aerodyn. 129, 69-102.

Blocken, B., Janssen, W.D., van Hooff, T., 2012. CFD simulation for pedestrian wind comfort and wind safety in urban areas: general decision framework and case study for the Eindhoven University campus. Environ. Model. Softw. 30, 15-34.

Carpman, N., 2011. Turbulence Intensity in Complex Environments and Its Influence on Small Wind Turbines (Ph.D. thesis). Uppsala University, Uppsala.

Cheng, Y., Lien, F.S., Yee, E., Sinclair, R., 2003. A comparison of large eddy simulations with a standard $k-\varepsilon$ Reynolds-averaged Navier-Stokes model for the prediction of a fully developed turbulent flow over a matrix of cubes. J. Wind Eng. Ind. Aerodyn. 91, 1301-1328.

Crespo, A., Manuel, F., Moreno, D., Fraga, E., Hernandez, J., 1985. Numerical analysis of wind turbine wakes. In: Proceedings of Delphi Workshop on Wind Energy Applications. Delphi, Greece, pp. 15-25.

Contrib setDiscreteFields, 2013. OpenFOAM Wiki. Web: $\langle$ http://openfoamwiki.net/ index.php/Contrib_setDiscreteFields $>$.

Durbin, P.A., 1996. On the $k-\varepsilon$ stagnation point anomaly. Int. J. Heat Fluid Flow 17, $89-90$.

Franke, J., Hellsten, A., Schlnzen, H., Carissimo, B., 2007. Best practice guideline for the CFD simulation of flows in the urban environment, COST Action 732. COST office, Brussels.

Gousseau, P., Blocken, B., van Heijst, G.J.F., 2013. Quality assessment of large-eddy simulation of wind flow around a high-rise building: validation and solution verification. Comput. Fluids 79, 120-133.

Grant, A., Johnstone, C., Kelly, N., 2008. Urban wind energy conversion: the potential of ducted turbines. Renew. Energy 33, 1157-1163.

Guidebook for Practical Applications of CFD to Pedestrian Wind Environment around Buildings, 2013. Architectural Institute of Japan, Tokyo. Web: 〈http:// www.aij.or.jp/jpn/publish/cfdguide/index_e.htm).

Jiménez, P.A., Dudhia, J., 2013. On the ability of the WRF model to reproduce the surface wind direction over complex terrain. J. Appl. Meteorol. Climatol. 52, $1610-1617$.

Kato, M., Launder, B.E., 1993. The modeling of turbulent flow around stationary and vibrating square cylinders. In: 9th Symposium on Turbulent Shear Flows, vol. 10. Kyoto, pp. 1-6.

Kim, J.-J., Baik, J.-J., 2004. A numerical study of the effects of ambient wind direction on flow and dispersion in urban street canyons using the RNG $k-\varepsilon$ turbulence model. Atmos. Environ. 38, 3039-3048.

Kono, T., Kogaki, T., 2013. Numerical investigation of wind conditions over a rectangular prism-shaped building for mounting small wind turbines. Wind Eng. $36(2), 111-122$.

Kooiman, S.J., Tullis, S.W., 2010. Response of a vertical axis wind turbine to time varying wind conditions found within the urban environment. Wind Eng. 34 389-401.

Launder, B.E., Kato, M., 1993. Modeling flow-induced oscillations in turbulent flow around a square cylinder. In: ASME Fluid Engineering Conference.

Ledo, L., Kosasih, P.B., Cooper, P., 2011. Roof mounting site analysis for micro-wind turbines. Renew. Energy 36, 1379-1391. 
Lu, L., Ip, K.Y., 2009. Investigation on the feasibility and enhancement methods of wind power utilization in high-rise buildings of Hong Kong. Renew. Sustain. Energy Rev. 13, 450-461.

Meng, T., Hibi, K., 1998. Turbulent measurements of the flow field around a highrise building. J. Wind Eng. 76, 55-64 (in Japanese).

Menter, F.R., 1994. Two-equation eddy-viscosity turbulence models for engineering applications. AIAA J. 32, 1598-1605.

Mouzakis, F., Morfiadakis, E., Dellaportas, P., 1999. Fatigue loading parameter identification of a wind turbine operating in complex terrain. J. Wind Eng. Ind. Aerodyn. 82, 69-88.

Ng, E., Yuan, C., Chen, L., Ren, C., Fung, J.C.H., 2011. Improving the wind environment in high-density cities by understanding urban morphology and surface roughness: a study in Hong Kong. Landsc. Urban Plan. 101, 59-74.

OpenFOAM, 2013. Web: 〈http://www.openfoam.com〉.

Panofsky, H., Dutton, J., 1984. Atmospheric Turbulence. Wiley, New York.

Pierik, J.T.G., Dekker, J.W.M., Braam, H., Bulder, B.H., Winkelaar, D., Larsen, G.C. et al., 1999. Wind energy for the next millennium. In: Proceedings of the European Wind Turbine Standards Il (EWTS-II). James and James Science Publishers, London.

Pope, S., 2000. Turbulent Flows. Cambridge University Press, Cambridge.

Rákai, A., Kristóf, G., Franke, J., 2014. Sensitivity analysis of microscale obstacle resolving models for an idealized Central European city center, Michel-Stadt. Q J. Hung. Meteorol. Serv. 118, 53-77.

Ramponi, R., Blocken, B., 2012. CFD simulation of cross-ventilation for a generic isolated building: impact of computational parameters. Build. Environ. 53, 34-48.

Ricciardelli, F., Polimeno, S., 2006. Some characteristics of the wind flow in the lower urban boundary layer. J. Wind Eng. Ind. Aerodyn. 94, 815-832.

Roache, P.J., 1998. Verification of codes and calculations. AIAA J. 36, 696-702.

Sagaut, P., 2006. Large Eddy Simulation for incompressible flows, third ed. Springer Berlin.

Santiago, J.L., Martilli, A., Martín, F., 2007. CFD simulation of airflow over a regular array of cubes. Part I: three-dimensional simulation of the flow and validation with wind-tunnel measurements. Bound.-Layer Meteorol. 122, 609-634.

Shao, J., Liu, J., Zhao, J., 2012a. Evaluation of various non-linear $k-\epsilon$ models for predicting wind flow around an isolated high-rise building within the surface boundary layer. Build. Environ. 57, 145-155.
Shao, J., Liu, J., Zhao, J., 2012b. Evaluation of various non-linear $k-\varepsilon$ models for predicting wind flow around an isolated high-rise building within the surface boundary layer. Build. Environ. 57, 145-155.

Shih, T.H., Zhu, J., Lumley, J.L., 1993. A realizable Reynolds stress algebraic equation model. NASA Tech Memo 105993.

SnappyHexMesh, 2013. OpenFOAM Wiki. Web: (http://openfoamwiki.net/index. php/SnappyHexMesh).

SnappyHexMeshDict, 2013. snappyWiki. Web: (https://sites.google.com/site/snap pywiki/snappyhexmesh/snappyhexmeshdict).

Sumner, J., 2012. Towards Improved RANS $k-\varepsilon$ Modelling of Turbulent Incompressible Flows for Wind Energy Applications (Ph.D. thesis). École de Technologie Supérieure, Université du Québec, Montreal.

Sumner, J., Watters, C.S., Masson, C., 2010. CFD in wind energy: the virtual, multiscale wind tunnel. Energies 3, 989-1013.

Toja-Silva, F., Colmenar-Santos, A., Castro-Gil, M., 2013. Urban wind energy exploitation systems: behaviour under multidirectional flow conditionsopportunities and challenges. Renew. Sustain. Energy Rev. 24, 364-378.

Tominaga, Y., Mochida, A., Murakami, S., Sawaki, S., 2008. Comparison of various revised it $\mathrm{k}-\epsilon$ models and LES applied to flow around a high-rise building model with 1:1:2 shape placed within the surface boundary layer. J. Wind Eng. Ind. Aerodyn. 96, 389-411.

Tsuchiya, M., Murakami, S., Mochida, A., Kondo, K., Ishida, Y., 1997. Development of a new $k-\varepsilon$ model for flow and pressure fields around bluff body. J. Wind Eng. Ind. Aerodyn. 67-68, 169-182.

van Hooff, T., Blocken, B., 2010. On the effect of wind direction and urban surroundings on natural ventilation of a large semi-enclosed stadium. Comput. Fluids 39, 1146-1155.

Whiteman, C.D., 2000. Mountain Meteorology: Fundamentals and Applications. Oxford University Press, Oxford.

Yakhot, V., Smith, L.M., 1992. The renormalization group, the $\varepsilon$-expansion and derivation of turbulence models. J. Sci. Comput. 7, 35-61.

Yap, C.J., 1987. Turbulent Heat and Momentum Transfer in Recirculating and Impinging Flows (Ph.D. thesis). Faculty of Technology, University of Manchester United Kingdom. 